\title{
Electroencephalogram (EEG) human stress level classification based on theta/beta ratio.
}

WEN, T.Y., BANI, N.A., MUHAMMAD-SUKKI, F. and MOHD-ARIS, S.A.

2020

(C) 2020 International Journal of Integrated Engineering 


\title{
Electroencephalogram (EEG) Human Stress Level Classification based on Theta/Beta Ratio
}

\author{
Tee Yi Wen ${ }^{1}$, Nurul Aini Bani ${ }^{1}$, Firdaus Muhammad-Sukki ${ }^{2}$, Siti Armiza Mohd \\ Aris $^{1^{*}}$
}

${ }^{1}$ Razak Faculty of Technology and Informatics, Universiti Teknologi Malaysia (UTM), Jalan Sultan Yahya Petra (Jalan Semarak), 54100 Kuala Lumpur, MALAYSIA

${ }^{2}$ Robert Gordon University,

Garthdee House, Garthdee Rd, Aberdeen AB10 7AQ, UNITED KINGDOM

*Corresponding Author

DOI: https://doi.org/10.30880/ijie.2020.12.06.020

Received 31 March 2020; Accepted 19 July 2020; Available online 30 July 2020

\begin{abstract}
Stress analysis by utilizing electroencephalography (EEG) device in conjunction with signal processing techniques has emerged as an important area of research and the efforts are being made on detecting and classifying stress level. Non-invasive EEG device is used in this study to collect brain signals and analyze the signals by applying the modified Welch's fast Fourier transform (FFT) algorithm to extract the power spectral density (PSD) of each frequency band and calculate the power ratio of Alpha to Beta and Theta to Beta. The analysis of the power ratio has further validated that the Theta/Beta power ratio can be used as feature of stress and thus, imported its dataset into $\mathrm{k}$-means clustering to divide the subjects into three categories. Lastly, the clustering model is fed into support vector machine (SVM) to classify three-level stress which are of low, moderate and high. The result has signified the feasibility and effectiveness of the three-level stress classification at overall classification accuracy of $90 \%$ by applying Theta/Beta power ratio of the brain signals as well as using SVM classifier. The outcome of the research suggests the proposed method can be used for the implementation of stress monitoring system.
\end{abstract}

Keywords: Stress, Electroencephalography, Power Ratio, Theta/Beta, Classification, Support Vector Machine

\section{Introduction}

Stress is a term easily used by everyone on daily basis as it occurs unavoidable from time to time. Modern life offers challenges and human beings are surrounded by stressful situations due to busy work schedule and deadline, relationship difficulties, family matters and financial problems. In Malaysia, the National Health and Morbidity Survey (NHMS) conducted in year 2017 reported that 1 in 5 adolescents aged 13 to 17 years old were depressed, 2 in 5 had anxiety and 1 in 10 suffered from stress [1]. Since all are equally at risk of experiencing stress, it is crucial to detect and monitor stress levels for early diagnosis in order to prevent possible future illnesses. Stress response originated from the brain but involving various biochemical and physiological effects. Stress activates primary hormonal response known as the hypothalamic-pituitary-adrenal (HPA) axis which ultimately increases the cortisol secretion in the adrenal cortex [2]. Elevation in cortisol levels indicates an increase in stress levels.

Furthermore, experimental studies have investigated that stress can be detected through changes in physiological signals including heart rate variability (HRV) [3], skin conductance [4] and brainwave frequency [5] which can be measured by using biomedical devices. There are studies have proven the positive association between the hormonal response and physiological variables used to measure stress [6-8]. The correlation of stress between salivary cortisol, HRV and brainwave frequency has been studied in this work [9]. Electrocardiogram (ECG) and electroencephalogram 
(EEG) devices were utilized to acquire HRV and brainwave frequency respectively. The positive relationship is explored as the result demonstrated the three physiological parameters are closely associated to chronic stress. The higher stress level manifests an increased salivary cortisol, lower HRV and higher activity in Beta frequency band. Brainwave activity reveals potential biomarker and has been proven able to represent important information of the brain function in responding to the stress [10].

Diverse brain imaging has grown into instruments to study stress assessment and importantly EEG has manifested the use of brain signals is impactful at distinguishing stress levels [11-13]. EEG signal processing and analysis assist the investigation of various aspects of human brain states [14-16] and problems associated with neurological disorders [17]. Stress pattern can be expressed by a decrease of power in Alpha band and an increase of power in Beta band [18] on prefrontal cortex (PFC) [19]. The PFC functioning is acknowledged as brain region which is susceptive to stress as stress exposure causes effects on this region [20]. The evolution of individuals' cognitive state in particular mental attentiveness [21] was studied in conjunction with the power ratio of Alpha/Beta. Apart from that, Theta/Beta ratio is initially established as an EEG marker to diagnose arousal deficit of attention deficit/hyperactivity disorder (AD/HD) [22]. However, this metric is linked to executive cognitive performances in healthy individuals [23].

The aim of this study is to analyze and examine the power ratio of Alpha/Beta and Theta/Beta as potential stress features based on the area of PFC. The EEG signals are first collected and pre-processed after which divide the frequency bands into Alpha, Beta, Theta and Delta. Fast Fourier transform (FFT) algorithm is then applied to extract the power spectral density (PSD) of the four frequency bands. Next, the PSD of Alpha, Beta and Theta frequency bands are averaged for the computation of Alpha/Beta ratio and Theta/Beta ratio in order to disclose the relationship between stress and the power ratio. The feasibility and validity of the Theta/Beta ratio are verified through the experimental results. The power ratio of Theta/Beta is decreased after exposed to the two stress stimuli which denote more stressful state and also implies the power ratio as potential stress feature for the development of stress classification model. The discovery of the study can be used for further analysis of EEG biomarkers of stress and the development of stress level classification system with the purpose of clinical intervention as well as disease prevention.

\section{Research Method}

Figure 1 shows the four experiment stages which are data collection, signal pre-processing, feature extraction and classification. Initially, the brain signals were acquired through EEG device followed by EEG signal pre-processing. Subsequently, four frequency bands were divided to extract their PSD for further calculation and finally, carry out the clustering and three-level stress classification with selected power ratio.

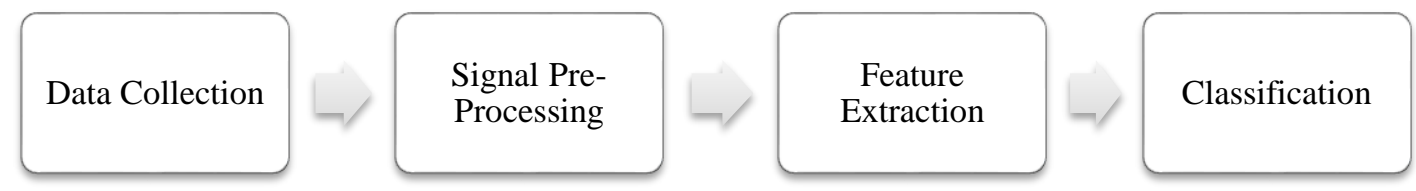

Fig. 1 - Overall process of the research work

\subsection{Data Collection}

The EEG device named g-MOBIlab is connected to five pieces of bipolar gold-plated EEG electrodes to amplify and obtain the EEG signals. The EEG signals were recorded at 5 scalp loci, comply with the international 10-20 electrodes placement system as referred to Figure 2. The monitored regions were prefrontal (Fp1 and Fp2). Fp1 was fixed on the left side of forehead and Fp2 on the right side of forehead, connecting to Channel 1 and Channel 2 respectively. In the meantime, both A1 and A2 have been attached to the earlobes for reference points. The g-MOBIlab performed with MATLAB-integrated Simulink model to record and transmit the EEG signals to the computer. The EEG device sampled the brain signals at $256 \mathrm{~Hz}$ and the impedance for all electrodes and cortex maintained below $5 \mathrm{k} \Omega$ was achieved by cleaning and removing the dirt and dead cells on skin with non-abrasive alcohol.

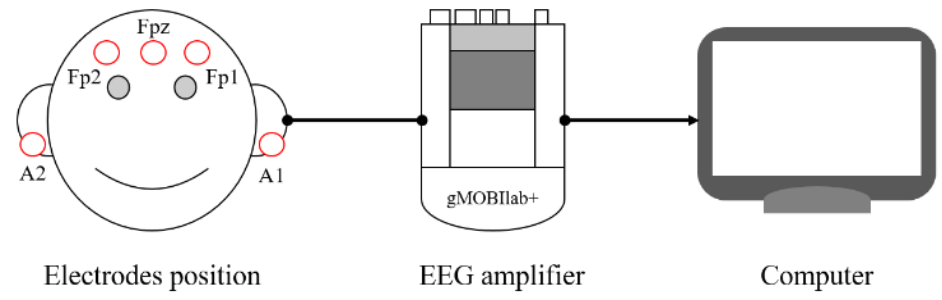

Fig. 2 - EEG measurement setup 
A sample group of 50 students from Universiti Teknologi Malaysia, Kuala Lumpur, Malaysia were recruited for this study. A clear verbal instruction was given to the subjects to familiarize them with the experimental tasks before commencement of the EEG recording as well as they were required to sign a consent form and fill in the demographic form prior to the experiment. The procedure of EEG signals recording for three different sessions is depicted in Figure 3. Initially, the subjects were given 5 to 10 minutes to relax in eyes-closed wakefulness. The first session was the EEG recording on eyes-closed at resting state condition for 3 minutes as the baseline measurement. This resting baseline session was to determine the difference in EEG power ratio between relax and the subsequent EEG recording of stressful conditions. Next, the subjects had to wear Samsung Gear VR device to experience a 360-degree horror video for 4 minutes during the second session of experiment. The EEG signals recording for another 3 minutes at eyes-closed resting condition was performed immediately after the VR video session ended as post-VR video. The EEG power ratio changes in between pre-VR video (baseline eyes-closed resting condition) and post-VR video was evaluated. Followed by the 20 minutes of IQ test as well as the 3 minutes of signal recording at eyes-closed resting condition after the IQ test session ended as post-IQ test. The EEG power ratio changes in between pre-IQ test (baseline eyes-closed resting condition) and post-IQ test was evaluated.

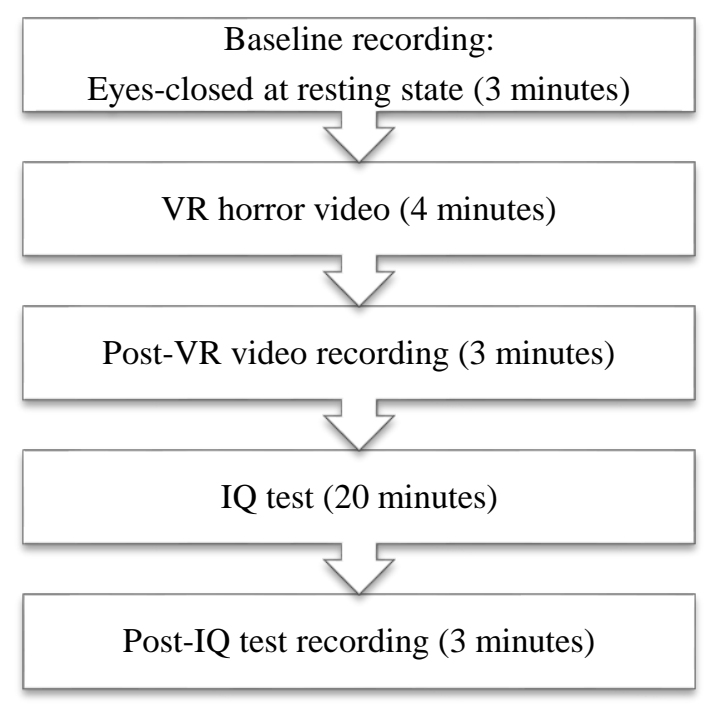

Fig. 3 - EEG experiment protocol

\subsection{Signal Pre-Processing}

The EEG signals were digitized at 256 samples per seconds and filtered by a notch filter at $50 \mathrm{~Hz}$ to eliminate the low frequency by power line using Simulink model before converting the EEG signals into desired file format. The EEG records where the areas contaminated by artefacts such as extreme values were rejected by setting a threshold value below than $-100 \mu \mathrm{V}$ and greater than $100 \mu \mathrm{V}$. The next step involved is designing the band-pass filter to cut off unwanted frequency and keeping the required frequency from $0.5 \mathrm{~Hz}$ to $30 \mathrm{~Hz}$ including Delta $(0.5-4 \mathrm{~Hz})$, Theta $(4-8 \mathrm{~Hz})$, Alpha $(8-13 \mathrm{~Hz})$ and Beta $(13-30 \mathrm{~Hz})$ by applying Hamming windowing function.

\subsection{Feature Extraction}

The spectral characteristics of the artifact-free EEG signals were computed via FFT algorithm from Welch method. MATLAB provides a built in command for the computation which is called pwelch function to obtain PSD by splitting the signals into segments, possibly overlapping, performing FFT, computing the magnitude of each segments and averaging these spectra. In this study, 1 minute was selected from the 3 minutes EEG signal as sample and divide the sample into 12 equal segments with equal duration windows in which the window length is 5 seconds each. Time sequence of every 1-minute sample was split up into 12 segments with a $50 \%$ overlap between each segment and each of which is windowed as well transformed using the FFT. Finally, the individual FFT responses or periodograms are averaged together and scaled to produce the spectral estimate which is the PSD ( $\mu$ V2) values of Alpha, Beta, Theta and Delta. The PSD according to Welch is described as follow [24]:

$$
P_{d}(f)=\frac{1}{M U}\left|\sum_{n=0}^{M-1} x_{d}(n) w(n) e^{-j 2 \pi f n}\right|^{2}
$$


Let $x_{d}(n)$ be the sequence, where $d=1,2,3, \ldots, L$ are the signal intervals and each interval length is $M . U$ is the normalization factor for the power in the window function while $w(n)$ is the windowed data, that is:

$$
U=\frac{1}{M} \sum_{n-0}^{M-1}|w(n)|^{2}
$$

The Welch power spectrum is the average over these modified periodograms can be represented as:

$$
P W e l c h(f)=\frac{1}{L} \sum_{i=0}^{L-1} P_{d}(f)
$$

Where PWelch is the periodogram of the EEG signal of each interval.

Besides calculating and averaging the PSD of each frequency bands, the relative power ratio of certain frequency bands was also computed since the power ratio of interest for this study are Alpha/Beta and Theta/Beta. Delta band was excluded in this study because this brainwave is commonly associated with deep sleeping state of a person. Hence, it was not expected to show high activity during the stressful condition. So as to achieve the power ratio of Alpha/Beta, the PSD value of Alpha was divided by the PSD value of Beta thenceforth to observe the power ratio between the resting baseline and the two stress sessions. The procedures as well applied to obtain the power ratio of Theta/Beta and observe the difference before and after the stimuli.

\section{Results and Analysis}

Above specified experiment stages include data collection, signal pre-processing and feature extraction have produced the power ratio of Alpha/Beta and Theta/Beta to proceed with the final experiment stage which is classification.

\subsection{Alpha/Beta Ratio vs Theta/Beta Ratio}

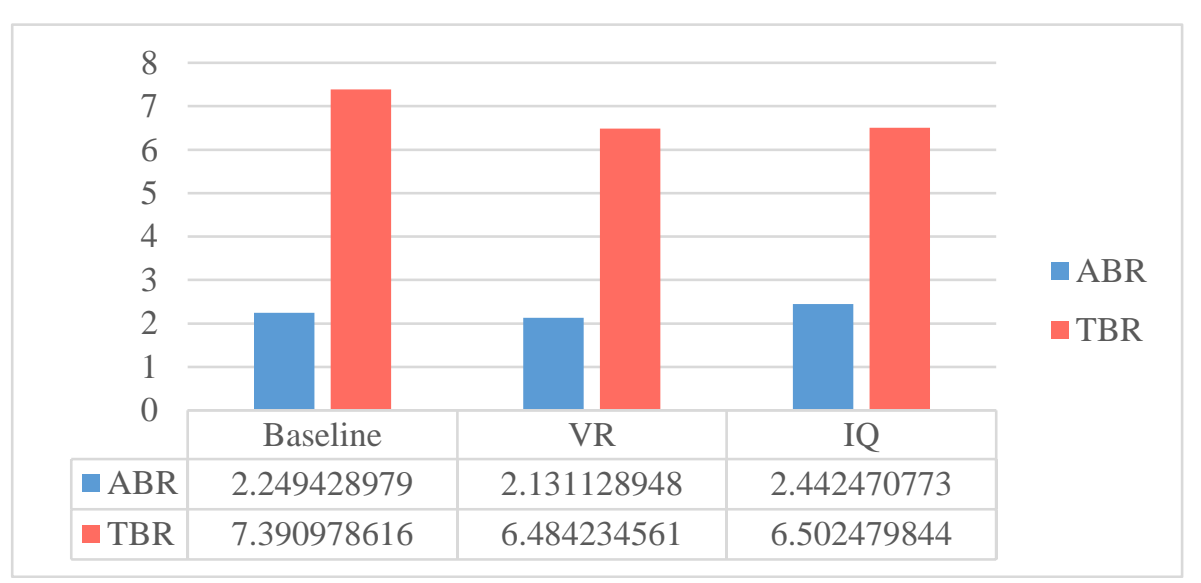

Fig. 4 - The comparison between Alpha/Beta Ratio (ABR) and Theta/Beta Ratio (TBR)

Alpha/Beta ratio and Theta/Beta ratio are analyzed for stress feature selection prior to classifying stress level. Alpha waves dominate when the brain is in an awaken but relaxed mental state whereas Beta waves dominate the brain in active, alert and stressful state. The declining value of Alpha/Beta ratio was reported in a study when the decision making requires higher engagement in cognitive processes [25]. Meanwhile in Figure 4, the first stressful condition which is VR video results in lower Alpha/Beta ratio compared to the baseline informs more demanding cognitive functions and higher level of stress. Consider the baseline the less stressing condition, the value of Alpha/Beta ratio is decreased after exposed to the first stimulus which indicates the VR stimulus as the more stressing one, a negative correlation between stress and the Alpha/Beta ratio is then unveiled. However, another level of stimulus which is IQ test appears higher ratio compared to the baseline signifies a lower level of stress. Therefore, the stress response generated from IQ test is positively associated with Alpha/Beta ratio. Nevertheless, the Theta/Beta ratio in Figure 4 unveils the baseline value has correlated negatively to both stress stimuli. Previous studies have reported that Theta waves are linked to the regulation of motivational and emotional processes under the brain activity [26] and the Theta/Beta ratio assists the study of emotional cognitive interactions and the relation to underlying biological functioning [27]. The decreased value of Theta/Beta ratio reflects increased frontal cortical control over subcortical with inhibition-driven affect, fear and higher anxiety which is 
compatible with the result as the stress response is associated with negative emotions. Concisely, the result has further validated that Theta/Beta ratio is rather applicable and advisable to be used as EEG stress feature to differentiate relax and stress. Thus, it is chosen for stress level classification.

\subsection{K-Means Clustering}

The dataset of Theta/Beta ratio is imported into clustering method to divide the subjects into different categories. Clustering is the process of dividing the entire data into groups based on the patterns in the data. K-means is used as the clustering process to group various objects based on their attributes in k number of groups. In order to cluster a given dataset, firstly specify $\mathrm{k}$, which is the number of clusters to be generated. $\mathrm{K}$ points are chosen randomly from existing data as cluster centers and each instance is calculated and assigned to its closet cluster center using Euclidean distance metric. Each instance is grouped among clusters based on minimum Euclidean distances. Next, the centroid (mean) for each cluster is calculated and used as a new cluster center. Following by the reassignment of all instances to the closet cluster center. The process iterates until till the algorithm converges or the cluster centers do not alter anymore. The objective function is described as follow [28]:

$$
J=\sum_{j=1}^{k} \sum_{i=1}^{x}\left\|X_{i}^{(j)}-C_{j}\right\|^{2}
$$

The algorithm aims at minimizing J function which is known as squared error function. K-means applies an iterative refinement method to produce its final clustering based on the dataset and the number of clusters defined by the user which is represented as variable $k$. The $\left\|X_{i}^{(j)}-C_{j}\right\|^{2}$ is a chosen distance measure or so called Euclidean distance between $X_{i}^{(j)}$ and the cluster center $C_{j}$. This is an indicator of the distance of the n data points from their respective cluster centers. In this study, the number of cluster is selected as 3 which are for low, moderate and high level of stress. Table 1 indicates the clustering assignment where the 17 subjects, 23 subjects and 10 subjects are clustered into the Low Stress, Moderate Stress and High Stress respectively.

Table 1 - Centroids value of the baseline, VR and IQ test

\begin{tabular}{ccccc}
\hline Attribute & $\begin{array}{c}\text { Full Data } \\
\text { (50 Subjects) }\end{array}$ & $\begin{array}{c}\text { Low Stress } \\
(\mathbf{1 7} \text { Subjects })\end{array}$ & $\begin{array}{c}\text { Moderate Stress } \\
\text { (23 Subjects) }\end{array}$ & $\begin{array}{c}\text { High Stress } \\
\text { (10 Subjects) }\end{array}$ \\
\hline Baseline & 7.391 & 5.0278 & 7.5831 & 10.9664 \\
VR & 6.4842 & 4.2556 & 6.3781 & 10.5171 \\
IQ & 6.5025 & 3.7483 & 7.2325 & 9.5056 \\
\hline
\end{tabular}

\subsection{SVM Classification}

Subsequently, the predicted stress level classification is resided in the suggested cluster. The clustering model above is then fed into support vector machine (SVM) to classify the stress level. The simple algorithm of SVM constructs an optimal hyperplane which divides data into classes with the maximum margin between the boundaries of the classes. The classification models are built to get a predicted result of data. In addition to that, model validation plays a crucial role to be an assurance of the accuracy and stability in order to estimate how accurately a predictive model will perform in practice. K-fold cross validation method is utilized in this case, the dataset is divided into k equal size subsets and the holdout method is repeated $\mathrm{k}$ times. Each time, one of the $\mathrm{k}$ subsets is tested and the other k-1 subsets are trained [29]. The present study has applied ten-fold cross validation as the stratified ten-fold is a suggested method for real-word datasets [30]. The ten-fold means nine folds are trained and one fold is tested. The process iterates until each subset is given a chance to be the test set once. The accuracy comes from the average of the k-tests accuracies.

Figure 5 has visualized the final result in which there are 5 out of 50 instances are incorrectly classified. The mistake is visualized in rectangle shape and the color represents its predicted cluster group. Whereas, the rest 45 instances in cross-shaped are correctly classified in their predicted cluster groups. The entire cross validation procedure is repeated 10 times to improve classification accuracy. 


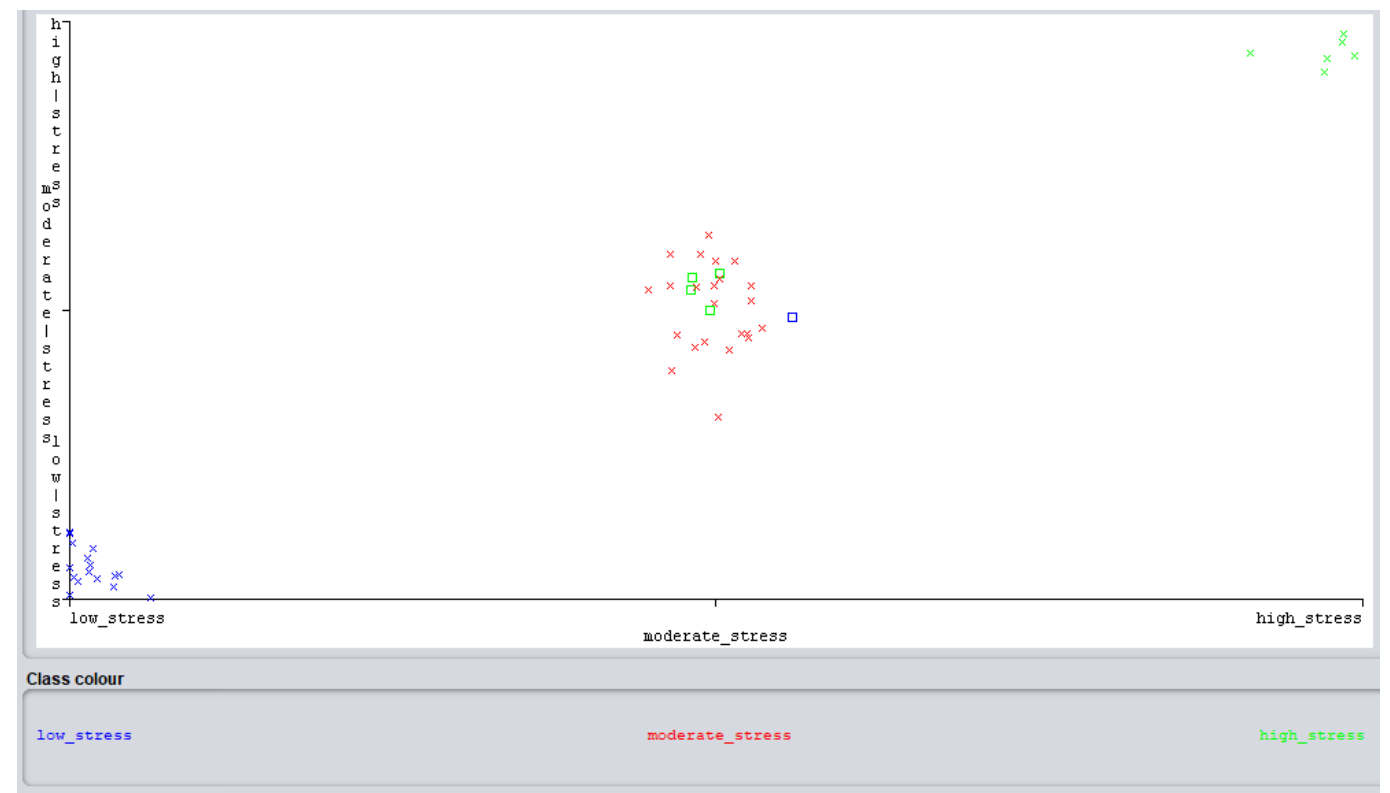

Fig. 5 - Stress level classification by low stress, moderate stress and high stress

Confusion matrix summarizes the prediction results and the performance of three-level stress classification using SVM. As shown in the Table 2, 16 subjects are correctly classified as low level of stress condition. This corresponds to $32 \%$ of all the 50 subjects. One subject is incorrectly classified as moderate stress and it corresponds to $2 \%$ of all the subjects. As for the moderate level of stress, 23 subjects which take up to $46 \%$ are correctly classified and the percentage of correct classification of the particular class is $100 \%$. Out of 10 highly stressed predictions, 6 subjects are correctly classified and the rest 4 subjects are wrongly classified into moderate stress condition. Based on the confusion matrix, the overall result of the three-level stress classification yields $90 \%$ of predictions are correct and $10 \%$ are wrong classifications.

Table 2 - Confusion matrix of SVM for three-level stress classification based on Theta/Beta ratio

\begin{tabular}{c|cccc|l}
\hline Confusion Matrix & \multicolumn{3}{|c|}{ Predicted Class } & \\
\hline & & $\mathrm{a}$ & $\mathrm{b}$ & $\mathrm{c}$ & Classified as \\
& $\mathrm{a}$ & $16(32 \%)$ & $1(2 \%)$ & $0(0 \%)$ & $\mathrm{a}=$ low_stress \\
Actual Class & $\mathrm{b}$ & $0(0 \%)$ & $23(46 \%)$ & $0(0 \%)$ & $\mathrm{b}=$ moderate_stress \\
& $\mathrm{c}$ & $0(0 \%)$ & $4(8 \%)$ & $6(12 \%)$ & $\mathrm{c}=$ high_stress \\
\hline
\end{tabular}

\section{Conclusion}

In this paper, the power ratio of slow wave over fast wave especially Theta/Beta ratio has been investigated and introduced as EEG stress feature as it can be used to reliably discriminate stress from resting state. The k-means clustering is used to divide the EEG power ratio of the subjects into subgroups for predicting the stress level. The subjects have been grouped successfully into their corresponding stress level using the value of Theta/Beta ratio. The clustering model is then used to develop a classification model and the result shows the EEG data can be classified well using SVM. The classifier is able to classify the into low, moderate and high level of stress state using Theta/Beta ratio as stress feature with $90 \%$ of accuracy. The proposed method produces a promising stress classification model and the future work will be involved the study to combine this power ratio with other EEG features to develop a more efficient and robust stress level classification system which is useful in the implementation of stress monitoring system or health indicator to provide better health care.

\section{Acknowledgement}

The authors would like to thank the Razak Faculty of Technology and Informatics, Universiti Teknologi Malaysia for the sponsorship of the study under research grant no.: Q.K130000.3556.06G43 and also to all collaborating partners.

\section{References}

[1] Institute for Public Health (IPH). (2017). National health and morbidity survey (NHMS) 2017: Adolescent Nutrition Survey 2017, Malaysia

[2] Smith, S. M., \& Vale, W. W. (2006). The role of the hypothalamic-pituitary-adrenal axis in neuroendocrine responses to stress. Dialogues in Clinical Neuroscience, 8(4), 383-395 
[3] Park, D., Lee, M., Park, S. E., Seong, J. K., \& Youn, I. (2018). Determination of optimal heart rate variability features based on SVM-recursive feature elimination for cumulative stress monitoring using ECG sensor. Sensors (Basel), 18(7), 15

[4] Villarejo, M. V., Zapirain, B. G., \& Zorrilla, A. M. (2012). A stress sensor based on galvanic skin response (GSR) controlled by zigbee. Sensors (Basel), 12(5), 6075-6101

[5] Hamid, N. H. A., Sulaiman, N., Murat, Z. H., \& Taib, M. N. (2015). Brainwaves stress pattern based on perceived stress scale test. 6th IEEE Control and System Graduate Research Colloquium, 135-140

[6] Sahoo, R., \& Sethi, S. (2015). Functional analysis of mental stress based on physiological data of GSR sensor. Advances in Intelligent Systems and Computing, 337, 109-117

[7] Allen, A. P., Kennedy, P. J., Cryan, J. F., Dinan, T. G., \& Clarke, G. (2014). Biological and psychological markers of stress in humans: Focus on the trier social stress test. Neuroscience \& Biobehavioral Reviews, 38, 94-124

[8] Hamer, M., O’Donnell, K., Lahiri, A., \& Steptoe, A. (2010). Salivary cortisol responses to mental stress are associated with coronary artery calcification in healthy men and women. European Heart Journal, 31(4), 424-429.

[9] Seo, S. H. (2016). Detection of chronic stress using bio-signals. Indian Journal of Science \& Technology, 9(46), 15

[10] Alonso, J. F., Romero, S., Ballester, M. R., Antonijoan, R. M., \& Mananas, M. A. (2015). Stress assessment based on EEG univariate features and functional connectivity measures. Physiological Measurement, 36(7), 1351-1365.

[11] Xia, L., Malik, A. S., \& Subhani, A. R. (2018). A physiological signal-based method for early mental-stress detection. Biomedical Signal Processing and Control, 46, 18-32

[12] Al-Shargie, F. M., Tang, T. B., Badruddin, N., \& Kiguchi, M. (2018). Towards multilevel mental stress assessment using SVM with ecoc: An EEG approach. Medical \& Biological Engineering \& Computing, 56(1), 125-136.

[13] Vanitha, V., \& Krishnan, P. (2016). Real time stress detection system based on EEG signals. Biomedical ResearchIndia, 27, S271-S275

[14] Mustafa, M., Nawas, K. A. A., Samad, R., Pebrianti, D., \& Abdullah, N. R. H. (2018). K-NN classification of brain dominance. International Journal of Electrical and Computer Engineering (IJECE), 8(4), 2494-2502

[15] Jahidin, A. H., Taib, M. N., Tahir, N. M., \& Ali, M. S. A. M. (2015). IQ classification via brainwave features: Review on artificial intelligence techniques. International Journal of Electrical and Computer Engineering (IJECE), 5(1), 84-91

[16] George, F. P., Shaikat, I. M., Hossain, P. S. F., Parvez, M. Z., \& Uddin, J. (2019). Recognition of emotional states using EEG signals based on time-frequency analysis and SVM classifier. International Journal of Electrical and Computer Engineering (IJECE), 9(2), 1012-1020

[17] Kidwai, M. S., \& Saeed, S. H. (2019). A novel approach for detection of neurological disorders through electrical potential developed in brain. International Journal of Electrical and Computer Engineering (IJECE), 9(4), 27512759

[18] Seo, S. H., \& Lee, J. T. (2010). Stress and EEG. Convergence and Hybrid Information Technologies, 410-426

[19] Al-Shargie, F. M., Kiguchi, M., Badruddin, N., Dass, S. C., Hani, A. F. M., \& Tang, T. B. (2016). Mental stress assessment using simultaneous measurement of EEG and fNIRS. Biomedical Optics Express, 7(10), 3882-3898

[20] Arnsten, A. F. T. (2009). Stress signalling pathways that impair prefrontal cortex structure and function. Nature Reviews Neuroscience, 10(6), 410-422

[21] Liu, N. H., Chiang, C. Y., \& Chu, H. C. (2013). Recognizing the degree of human attention using EEG signals from mobile sensors. Sensors (Basel), 13(8), 10273-10286

[22] Lubar, J. F. (1991). Discourse on the development of EEG diagnostics and biofeedback for attentiondeficit/hyperactivity disorders. Biofeedback and Self-Regulation, 16(3), 201-225

[23] Clarke, A. R., Barry, R. J., Karamacoska, D., \& Johnstone, S. J. (2019). The EEG Theta/Beta ratio: A marker of arousal or cognitive processing capacity?. Applied Psychophysiology and Biofeedback, 44(2), 123-129

[24] Subha, D. P., Joseph, P. K., Acharya, U. R., \& Lim, C. M. (2010). EEG signal analysis: A survey. Journal of Medical Systems, 34(2), 195-212

[25] Salma, N., Mai, B., Namuduri, K., Mamun, R., Hashem, Y., \& Takabi, H. et al. (2017). Using EEG signal to analyze IS decision making cognitive processes. Information Systems And Neuroscience, 211-218

[26] Knyazev, G. G. (2007). Motivation, emotion, and their inhibitory control mirrored in brain oscillations. Neuroscience \& Biobehavioral Reviews, 31(3), 377-395

[27] Putman, P., van Peer, J., Maimari, I., \& van der Werff, S. (2010). EEG Theta/Beta ratio in relation to fear-modulated response-inhibition, attentional control, and affective traits. Biological Psychology, 83(2), 73-78

[28] Kalas, M. S., \& Momin, B. F. (2016). Stress detection and reduction using EEG signals. International Conference on Electrical, Electronics, and Optimization Techniques (ICEEOT), 471-475

[29] Hastie, T., Tibshirani, R., \& Friedman, J. (2009). The elements of statistical learning: Data mining, inference, and prediction. Springer

[30] Kohavi, R. (1995). A study of cross-validation and bootstrap for accuracy estimation and model selection. International Joint Conference on Articial Intelligence, 2, 1137-114 